\title{
SCPA: A Segemented Carry Prediction Approximate Adder Structure
}

\author{
Hao Liu ${ }^{1}$, Ming-Jiang Wang ${ }^{1}$, and Ming Liu ${ }^{2}$
}

\begin{abstract}
Approximate computing has excellent result in error-tolerant applications sacrificing computational accuracy for better performance in the area, speed, and power consumption. As the most basic operation, addition is used in a large number of applications in various occasions. Therefore it is of great importance to optimize the performance of addition computation. In this paper, a segemented carry prediction adder (SCPA) structure is proposed, which splits the long carry chain into several short chains for parallel computation. The design parameters are diversified by adjusting the size of the blocks and the prediction depth of each subadditive to achieve different levels of performance. Flexible parameter tuning allows different design goals to be achieved based on specific performance requirements, which makes SCPA a useful design guideline for approximate adders. The error performance of SCPA is mesured by MRED, NMRED, $\mathrm{ER}$, and other indicators and significantly has the best statiscal performace compared to similar designs. The proposed design is synthesized under TSMC $65 \mathrm{~nm}$ process, and the result shows that the SCPA has a very nice accuracy-power tradeoff under 8-bit and 16-bit condition.

key words: Approximate computing, adder, hardware design. Classification: Integrated circuit(logic)
\end{abstract}

\section{Introduction}

With the advent of big data and artificial intelligence, the demand for computing power is increasing at this stage in various fields, and improving computing efficiency has become a new challenge. Achieving the desired level of performance can be a challenge due to limited power budgets. In binary arithmetic, adders are considered fundamental blocks of digital systems at the microarchitecture's level. Therefore the performance improvement of adders is of great importance [1].

Approximate computing technique is a new design approach that sacrifices computational accuracy to increase computational speed and reduce circuit area. It can be used for multimedia and image processing, digital signal process-

\footnotetext{
${ }^{1}$ Faculty of Electronics and Information Engineering, Harbin Institute of Technology, Shenzhen 518055, GuangDong, China

${ }^{2}$ Sino-German School, Shenzhen Institute of Information Technology, Shenzhen 518055, GuangDong, China

a) gabriel.carson.law @ outlook.com

b) lm_hit_1986@126.com
}

DOI: $10.1587 /$ elex.18.20210335

Received August 12, 2021

Accepted August 25, 2021

Publicized September 15, 2021 ing, wireless communication, data mining, and other errorresilient applications to achieve more energy reduction and performance improvement at the cost of acceptable accuracy loss [2]. Various kind of approximate computational techniques have been proposed at different software/hardware levels, and many approximate adder designs solving system performance bottlenecks have been proposed [3-18], which are mostly through hardware manipulation, logic simplification, and voltage overscaling to achieve energy and speed gains [19]. The design idea of approximate computation can greatly reduce the delay and circuit area of addition computation and significantly improve the performance.

In this paper, we propose a high-speed, low-consumption segmented carry prediction adder architecture (SCPA). The adder is separated into parallel computational blocks with independent carry inputs generated by the carry prediction logic. The length of the critical path is reduced by truncating the carry-propagation chain. This approach minimizes the delay of long chains to the computational latency of the sub-adder block. In addition, the size of the sub-adder and the prediction depth are completely independent parameters, which significantly increases the flexibility of the design, allowing different adder structures to be obtained by tuning the parameters. By adjusting two key parameters, the SCPA structure can be varied to many similar adder designs, including RAP-CLA, SARA etc. The proposed adder structure is compared with some advanced approximate adders to analyze its performance. And we use the DCT and inverse DCT transforms in image processing to verify the practical application of the proposed design.

The rest of this paper is organized as follows. Section II reviews some related work. Section III presents the details of the proposed segmented carry prediction adder. In Section IV, the approximate adder's error performance and hardware consumption are verified and analyzed comparing with serveral adder structures. Finally, we get a conclusion in Section V.

\section{Background and Related Work}

In approximate computing circuits, there are two general methods to increase speed and reduce power. The first approach is to use voltage overscaling (VOS) technique in CMOS circuits to save energy. The arithmetic accuracy can 
be tuned at runtime using dynamic voltage and frequency scaling methods to obtain the best accuracy-power tradeoff. The benefits of run-time accuracy tuning are demonstrated in [20], but the approximation is achieved by voltage overscaling, and errors mainly occur in the timing critical paths associated with the most significant bits; thus, the errors tend to be large. The second approach improves the circuit by simplifying and adapting the algorithm itself and redesigning the hardware from the point of view of the hardware structure, which is more stable and controllable, with better error performance. So a brief review of the redesigned approach is presented in this section.

A reconfigurable approximate carry look-ahead adder (RAPCLA) is proposed in [21]. The structure of this adder is based on some modifications to the conventional CLA structure. The ability to switch between approximate and accurate modes makes it suitable for error-resistant and accurateneed applications. In the RAP-CLA, fixed-size overlapping sub-blocks are used to compute carry bits, which has lower delay, power, and area in the approximate mode than in the accurate CLA.

Reference [22] proposed an approximate adder HEAA, which consists of accurate and approximate parts. The approximate part is somewhat similar to the inaccurate part of LOA [23]. However, the approximate part of HEAA includes an additional multiplexer. The AND result of the most significant input bit pair of the approximate part is used as the selection signal of the multiplexer and the incoming input of the accurate part.

Paper [24] presents an approximate adder HOERAA, also consisting of an accurate part and approximate part. The accurate part is implemented using a conventional adder. For the approximate part, the most significant sum bit is generated by a specific logic. The next significant sum bit is generated by the OR result of the corresponding input bit pair, and the rest of the sum bits are assigned a constant of 1.

Reference [25] proposed a hardware-optimized approximate adder with a normal error distribution called HOAANED. Similar to HOERAA, the adder structure is truncated and divided into two parts. The high-weight part does not use approximate techniques to guarantee the correctness of the computational results. The low-weight part is set to a fixed value so that only approximate results are obtained. This kind of design pattern gives better statistical metrics but cannot calculate input data for small values.

In [16], a simple accuracy reconfigurable adder(SARA) is proposed. In this structure, the adder is divided into nonoverlapping sub-adders. For the first and last of the subadders, two improved full adders are used. A simple carry prediction mechanism is used in the design, making the circuit complexity smaller and introducing a loss of accuracy. In [3], a reconfigurable approximate adder is proposed that utilizes a dual-mode full adder. The dual-mode full adder contains the approximate full adder and an accurate con- ventional full adder. The output of the dual-mode full adder is selected by two multiplexers. Since each dual-mode full adder uses two additional multiplexers, this approach has a considerable delay, area, and power usage in the accurate operating mode.

An accuracy-configurable adder(ACA) is proposed in [27] that operates in both accurate and approximate modes. The core of the ACA is an approximate adder, and in accurate mode an error detection and correction (EDC) unit is used to produce accurate results. In the approximate mode, this structure utilizes a large number of sub-adders, resulting in a large use of power and area. In the accurate mode, the adder uses additional cycles for correction, which causes a large delay in addition.

In [28], a segmented approximate adder is proposed which improves the accuracy by carry prediction structure. In addition, an error-correction unit is used to reduce the error of the adder. The delay of this adder is larger than that of the conventional CLA with and without the error-reducing unit. Note that by increasing the segment size, the delay of this adder becomes much larger than the conventional CLA.

A low energy consumption block-based carry speculative approximate adder(BCSA) is proposed in [29]. Its structure is based on partitioning the adder into some non-overlapped addtion blocks Besides, it also has an error detection and recovery mechanism.

Dalloo [30] proposed an approximate adder OLOCA by modifying the LOA architecture. In the approximate part of the LOA, only the OR gate is used to combine the two most significant input bit pairs to produce their two most significant sum bits. The other sum bits are assigned a constant 1 . There is a significant statistical performance improvement compared to the truncated adder OLOCA with directly fixed low bits.

\section{Hardware Structure}

The performance bottleneck of conventional adders is that the length of the carry chain determines the critical path. In accurate adders, critical paths can be shortened by removing carry correlations but correspondingly increase the area consumption of the circuit,such as carry look-ahead adders. After relaxing the requirement of computational accuracy, approximate addition circuits can effectively shorten the critical path delay of a circuit and reducing the circuit area (or unchanged) at the expense of computational accuracy to improve the computing performance.

Common approximate adder structure can be divided into segmented adders and truncated adders. Segmented adders are primarily based on the spatiality of the carry information. Long-bit chain is sperated to to short chains that can be calculated in parallel. Carry information too far apart will be ignored, which can increase the speed of calculation.

Truncation adders divide conventional adders into accurate and approximate parts. Because the low part of the data has a smaller weight, the calculation of the low part is abandoned 
in the truncation adder. This way, the circuit complexity can be extremely reduced. Moreover, it can get suitable statistical performance. However, this approach will crash when data entered a small range. Besides, under the same conditions, the speed of the truncation adder is slower than that of the segmented adder.

The generation of the predicted carry bit is the core problem of the segmented adder. Consider the highest bit operation of the $i$ ith sub-adder $a_{i}, b_{i}, c_{i}$. In the conventional CLA design, the carray propagation equation is given as follows:

$$
\begin{aligned}
g_{i} & =a_{i} b_{i} \\
p_{i} & =a_{i} \oplus b_{i} \\
c_{i} & =g_{i}+p_{i} c_{i-1}
\end{aligned}
$$

Iterating the above equation yields the iterative expression for the carry generation logic in accurate addition.

$$
c_{i+1}=g_{i}+g_{i-1} p_{i}+\cdots+g_{0} \prod_{j=1}^{i} p_{j}+c_{i n} \prod_{j=0}^{i} p_{j}
$$

We can split the Eq. 1 into the following two parts in the approximate calculation logic to simplify the logic.

$$
c_{i+1}=\left(\sum_{j=i-w+1}^{i} g_{j}\left(\prod_{k=j+1}^{i-1} p_{k}\right)\right)+\left(\sum_{j=0}^{i-w} g_{j}\left(\prod_{k=j+1}^{i-1} p_{k}\right)+c_{i n} \prod_{j=0}^{i} p_{j}\right)
$$

If the second term is removed, then the calculation process will be simplified. But the corresponding carry bit will be inaccurate, and the smaller $w$ is, the lower the computational accuracy will be.

In the proposed structure, we split the addition operation of $n$ bit data into $m$ short-chain additions with sub-adders for parallel computation. Then the length of each sub-adders is $l=n / m$. The sub-adders are computed in accurate addition mode and can be implemented using CLA or RCA. Each sub-adder has independent input of carry bit, which is derived by pre-addition logic and carry prediction logic. The overall structure of the adder is shown in the Fig. 1. Unlike the general segmented adder, the prediction depth and the size of the sub-adders are not related in the proposed adder structure, and the width of each carry prediction block can be any suitable value even larger than the size of the sub-adders. In Fig. 1 we use different color bar to indicate the prediction depth, if the depth is larger than the block size, which means $l<w$, the prediction block will overlapped each other, just as the shadow area shown in the figure. Furthermore, the value of $w$ is not necessarily the same. This design idea integrates the overlapping block design and segmented adder, which is more flexible and can get a more suitable adder design according to the accuracy requirements. The pre-addition logic gives two outputs, $P$ and $G$ vectors composed of $p_{i}$ and $g_{i}$ we've talked above, and both can be re-used in the following addition operations. The carry prediction logic implements the prediction process just as the firt term of Eq. 3 This approach ensures the independence among subadder, shortens the critical path of the adder, and improves

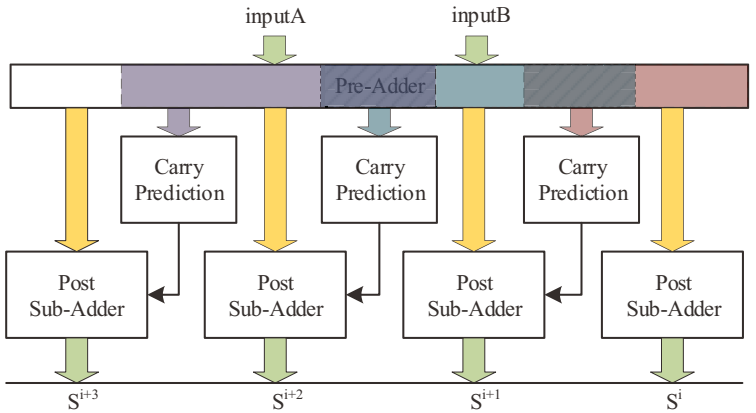

Fig. 1. Top level structure of SCPA structure

the computation speed. However, since the carry prediction logic does not take into account all the input information. There is an inevitable loss in computational accuracy.

\section{Result and Analysis}

\subsection{Error analysis}

The error performance is very critical in the approximation calculation and determines the reliability of the approximated design. In this section, the error performance of SCPA will be illustrated by comparing it with similar methods including SARA, RAP-CLA, and BCSA.

In the approximation calculation, the common error metrics include MED, ER, MRED, NMED, etc. MED stands for Mean Error Distance, and is the average of the absolute value of the difference between the approximate and true results. This is a very intuitive and basic indicator. However, because its value is not strongly correlated with the input data width, there is no way to truly reflect the accuracy performance of the calculation performace. Therefore, in this paper, NMED and MRED are used as main error measures.If we use D to indicate the max error distance(ED), then NMED is calculated as follows:

$$
\mathrm{NMED}=\frac{\mathrm{MED}}{D}=\frac{1}{2^{2 n}} \sum_{i=1}^{2^{2 n}} \frac{\mathrm{ED}_{\mathrm{i}}}{D}
$$

Mean relative error distance (MRED) is the average of the relative error, which better reflects the confidence level of the measurement. It is calculated as follows if we use $\mathrm{S}$ to stand for the standard result.

$$
\operatorname{MRED}=\frac{1}{2^{2 n}} \sum_{i=1}^{2^{2 n}} \frac{\left|\mathrm{ED}_{i}\right|}{S_{i}}
$$

The error rate(ER) is a very important indicator of the approximate adder. In combination with other indicators, the stability of the whole design can be evaluated, among which the widely used is the acceptable rate(AR). AR indicates the tolerance to error, that is, any result with a relative error distance less than a certain value can be considered as the correct result. Under this premise, the value of ER will be taken with some changes. Table I shows the comparison of 
the AR cases of the four adders. For the convinience of comparison, the prediction depth $d$ and block size $l$ of SCPA are both assumed to the same value as $w$ in the follwing discussion. From the table it is clear that SCPA has the best AR

Table I. ER comparation with 16bit data, $w=4$

\begin{tabular}{llllllll}
\hline RED $(\%)$ & $\leq 5 \%$ & $\leq 10 \% i$ & $\leq 20 \%$ & $\leq 30 \%$ & $\leq 35 \%$ & $\leq 50 \%$ & $\leq 100 \%$ \\
\hline RAP-CLA & $93.71 \%$ & $95.38 \%$ & $97.04 \%$ & $97.56 \%$ & $98.37 \%$ & $99.47 \%$ & $100 \%$ \\
\hline BCSA & $93.80 \%$ & $98.21 \%$ & $99.61 \%$ & $99.67 \%$ & $99.88 \%$ & $99.91 \%$ & $100 \%$ \\
\hline SARA & $91.68 \%$ & $97.91 \%$ & $99.53 \%$ & $99.71 \%$ & $99.87 \%$ & $99.95 \%$ & $100 \%$ \\
\hline SCPA & $97.89 \%$ & $99.47 \%$ & $99.89 \%$ & $99.94 \%$ & $99.97 \%$ & $99.99 \%$ & $100 \%$ \\
\hline
\end{tabular}

performance, even in the case of strict RED conditions also performs well.

Table II shows the performance comparison of four adders, RAP-CLA,SARA, BCSA, and SCPA, for different design parameters. 8bit data has a full recovery size of $2^{8} \times 2^{8} \times 2=$ $131,072(a \times b \times c)$, while 16bit and 32bit dataset both contain $1,000,000$ random data. From the table, we can learn that with the grow of $w$ the NMED, MRED and ER will decrease, and under the same $w$, the proposed design significantly has the best statistical performance.

Fig. 2 shows the logarithmic distribution of the relative error distances for the four cases. It can be seen that SARA, BCSA, and SCPA have similar relative error distribution. This is because the incoming prediction blocks in the RAP-CLA adder are highly overlapping. The prediction operation is performed for each bit, all of which may generate errors, and thus the error distribution is more complex. The other three approximate adders are all based on a blockwise adder without crossover. Therefore the location of error generation is distributed at the intersection of sub-adders. Since the parameters set in the picture are 16bit with $w=4$, thus the error will be distributed at three levels. Since the minimum error value is 1 , the relative error becomes larger when input value is close to zero. It is clear from the figure that the error point of SCPA is more sparse.

\subsection{Circuit consumption analysis}

The synthesis was done user the TSMC $65 \mathrm{~nm}$ technology libaray. Table III shows the hardware performance compar-

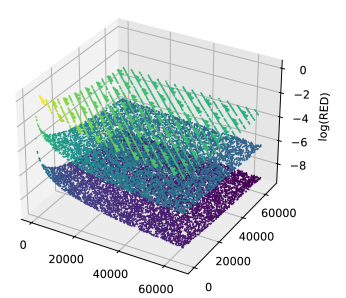

(a) BCSA

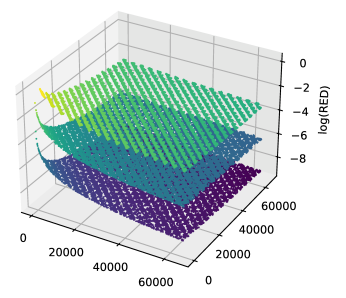

(c) SARA

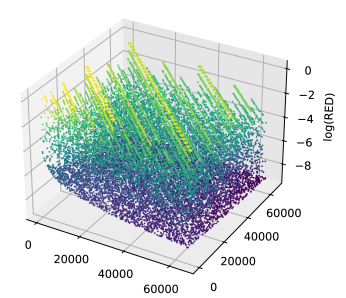

(b) RAP-CLA

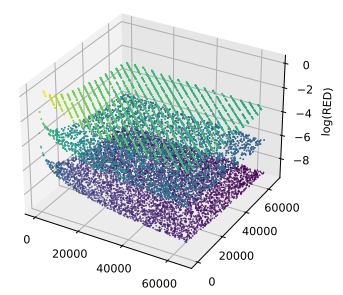

(d) SCPA
Fig. 2. The $\log (\mathrm{RED})$ for 16 -bit data, with $w=4$

ison of the four adders with different parameters for the 16-bit case. It is clear that RAP-CLA has the best speed performance, but the corresponding area is the largest. SARA has a smaller area and computational latency with CLA structure due to its simple carry prediction mechanism. The proposed SCPA has a moderate area and power consumption level but larger computational latency, which is a sacrifice made for better statistical performance.

Fig. 3 shows the combined performance of the four designs in terms of the power-delay product (PDP) as the horizontal coordinate. The dots in the figure represent the various approximate adders with different parameters. the SCPA structure has a better accuracy-power tradeoff when computing 8-bit and 16-bit data. The computational performance still dominates in the face of 32-bit data, but the computational power consumption of the SCPA is much higher compared to other adders due to the much higher computational complexity.

Table II. Error metrics comparation

\begin{tabular}{|c|c|c|c|c|c|c|c|c|c|c|}
\hline \multirow[b]{2}{*}{ Adder } & \multirow[b]{2}{*}{ W } & \multicolumn{3}{|c|}{ 8bits } & \multicolumn{3}{|c|}{ 16bits } & \multicolumn{3}{|c|}{ 32bits } \\
\hline & & $\begin{array}{l}\text { NMED } \\
\left(10^{-3}\right)\end{array}$ & $\begin{array}{l}\text { MRED } \\
\left(10^{-3}\right)\end{array}$ & $\begin{array}{l}\text { ER } \\
(\%)\end{array}$ & $\begin{array}{l}\text { NMED } \\
\left(10^{-3}\right)\end{array}$ & $\begin{array}{l}\text { MRED } \\
\left(10^{-3}\right)\end{array}$ & $\begin{array}{l}\text { ER } \\
(\%)\end{array}$ & $\begin{array}{l}\text { NMED } \\
\left(10^{-3}\right)\end{array}$ & $\begin{array}{l}\text { MRED } \\
\left(10^{-3}\right)\end{array}$ & $\begin{array}{l}\text { ER } \\
(\%)\end{array}$ \\
\hline \multirow{3}{*}{ BCSA } & 2 & 132.81 & 55.41 & 38.09 & 131.61 & 55.14 & 66.68 & 130.96 & 55.33 & 90.28 \\
\hline & 4 & 85.94 & 7.90 & 8.59 & 83.31 & 8.08 & 23.80 & 84.16 & 8.24 & 47.14 \\
\hline & 8 & 0.00 & 0.00 & 0.00 & 64.20 & 0.35 & 6.42 & 62.84 & 0.34 & 17.94 \\
\hline \multirow{3}{*}{ SARA } & 2 & 190.48 & 75.67 & 52.73 & 187.05 & 74.89 & 80.92 & 187.37 & 75.25 & 96.90 \\
\hline & 4 & 250.00 & 20.73 & 25.00 & 234.55 & 20.67 & 56.83 & 235.93 & 20.91 & 85.41 \\
\hline & 8 & 0.00 & 0.00 & 0.00 & 251.16 & 1.34 & 25.12 & 248.55 & 1.33 & 57.74 \\
\hline \multirow{3}{*}{ RAP-CLA } & 2 & 111.11 & 73.21 & 40.23 & 109.24 & 72.16 & 67.26 & 108.84 & 72.51 & 90.24 \\
\hline & 4 & 31.25 & 19.08 & 7.81 & 30.35 & 19.03 & 19.34 & 30.17 & 19.08 & 38.56 \\
\hline & 8 & 0.00 & 0.00 & 0.00 & 1.93 & 1.17 & 0.89 & 2.01 & 1.23 & 2.44 \\
\hline \multirow{3}{*}{ SCPA } & 2 & 117.65 & 39.09 & 30.08 & 117.46 & 38.56 & 55.11 & 116.67 & 38.73 & 81.53 \\
\hline & 4 & 31.25 & 2.65 & 3.12 & 31.10 & 2.59 & 8.89 & 30.98 & 2.61 & 19.50 \\
\hline & 8 & 0.00 & 0.00 & 0.00 & 2.07 & 0.01 & 0.21 & 2.02 & 0.01 & 0.58 \\
\hline
\end{tabular}


Table III. Hardware comparison

\begin{tabular}{ccccc}
\hline Method & $\mathrm{W}$ & $\begin{array}{c}\text { Area } \\
\left(\mu \mathrm{m}^{2}\right)\end{array}$ & $\begin{array}{c}\text { Power } \\
(\mu W)\end{array}$ & $\begin{array}{c}\text { Delay } \\
(n s)\end{array}$ \\
\hline \multirow{3}{*}{ SARA } & 2 & 268.8 & 31.49 & 0.25 \\
\cline { 2 - 5 } & 4 & 347.2 & 42.89 & 0.37 \\
\cline { 2 - 5 } RAP-CLA & 8 & 565.6 & 73.72 & 0.59 \\
\cline { 2 - 5 } & 2 & 243.2 & 27.85 & 0.22 \\
\hline \multirow{3}{*}{ BCSA } & 4 & 358.4 & 43.38 & 0.31 \\
\cline { 2 - 5 } & 8 & 698.8 & 90.78 & 0.68 \\
\hline \multirow{3}{*}{ SCPA } & 4 & 434.0 & 41.43 & 0.30 \\
\cline { 2 - 5 } & 8 & 484.0 & 52.40 & 0.41 \\
\cline { 2 - 5 } & 2 & 668.8 & 82.11 & 0.89 \\
\cline { 2 - 5 } & 4 & 385.6 & 33.14 & 0.39 \\
\hline
\end{tabular}

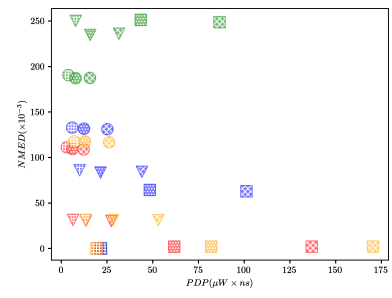

(a) NMED

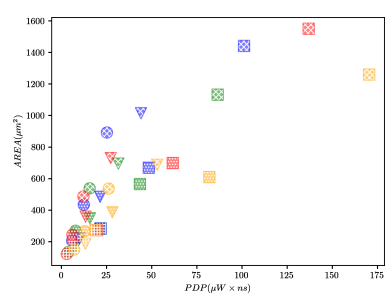

(c) Area

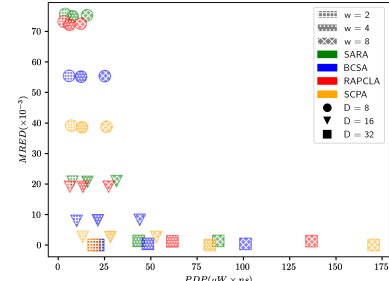

(b) MRED

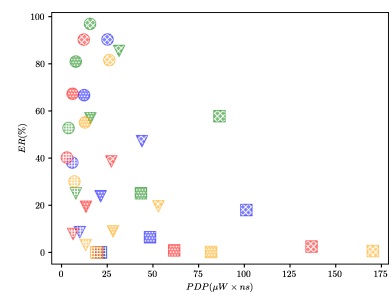

(d) ER
Fig. 3. Performance and consumption trade-off

\subsection{Application}

In order to verify the use of scpa in practical applications, we use the dct transform and inverse dct transform, which are common in image processing, as the measures for evaluating the performance of scpa. The test dataset uses 10 grayscale images commonly used in image processing. The results are shown in the table IV. SCPA has the highest average PSNR and the lowest MSE.

\begin{tabular}{lllllllll}
\multicolumn{8}{c}{ Table IV. DCT test } \\
\hline \multirow{2}{*}{ Figure } & \multicolumn{2}{c}{ BCSA } & \multicolumn{2}{c}{ RAPCLA } & \multicolumn{2}{c}{ SARA } & \multicolumn{2}{c}{ SCPA } \\
\cline { 2 - 9 } & MSE & PSNR & MSE & PSNR & MSE & PSNR & MSE & PSNR \\
\hline cameraman & 323.15 & 23.04 & 4076.58 & 11.68 & 7039.56 & 9.66 & 176.27 & 25.67 \\
\hline house & 341.25 & 22.77 & 8538.54 & 8.82 & 7149.71 & 9.55 & 250.02 & 24.12 \\
\hline jetplane & 386.52 & 21.40 & 6679.81 & 9.85 & 6866.77 & 8.90 & 95.76 & 27.46 \\
\hline lake & 435.05 & 21.22 & 5054.38 & 10.24 & 6772.56 & 9.30 & 64.71 & 29.49 \\
\hline lena & 422.21 & 21.42 & 3179.45 & 12.58 & 6883.91 & 9.29 & 65.84 & 29.49 \\
\hline livingroom & 417.57 & 21.92 & 3253.23 & 13.01 & 6837.73 & 9.78 & 66.33 & 29.91 \\
\hline mandril & 453.38 & 20.52 & 4629.17 & 10.43 & 6879.92 & 8.71 & 87.16 & 27.68 \\
\hline peppers & 370.79 & 21.20 & 2980.40 & 12.15 & 6845.46 & 8.53 & 89.51 & 27.37 \\
\hline walkbridge & 491.36 & 21.22 & 2428.94 & 14.28 & 6793.89 & 9.81 & 54.92 & 30.73 \\
\hline woman & 326.99 & 22.99 & 6074.56 & 10.30 & 6858.76 & 9.77 & 121.40 & 27.29 \\
\hline average & 396.83 & 21.77 & 4689.51 & 11.33 & 6892.83 & 9.33 & 107.19 & 27.92 \\
\hline
\end{tabular}

\subsection{Self Parameter Comparison}

Two important parameters of SCPA, $l$ and $w$, can be adjusted as needed. And due to the independence of the prediction

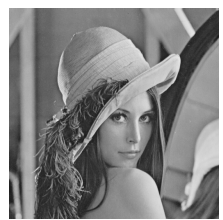

(a) Original

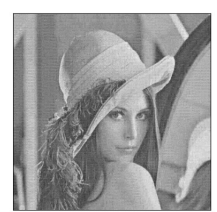

(b) BCSA

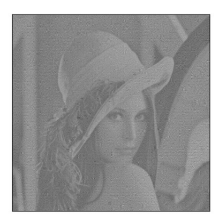

(c) RAP-CLA

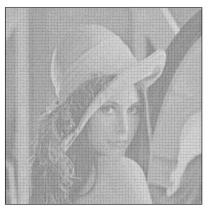

(d) SARA

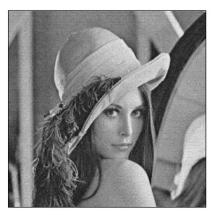

(e) SCPA
Fig. 4. DCT test result example with image 'lena'

blocks, the prediction logic can also be tuned. Thus SCPA is highly flexible. We can even extend other approximate adders as a special case of SCPA. If we fix $l$ to 1 and change $w$ arbitrarily. then we get RAP-CLA. if $w$ is fixed to 1 and $l$ is taken to be equal, then we get BCSA. In this section we use an example to discuss the performance in SCPA under different $w$. The simulation and synthesis result are shown in Table $\mathrm{V}$, in which $w_{3}, w_{2}, w_{1}, w_{0}$ means the predciton depth from the highest weight sub-adder to the lowest ones using 16-bit data and $l=4$.

Table V. Self Parameter Comparison

\begin{tabular}{lllllll}
\hline$w_{3} w_{2} w_{1} w_{0}$ & $\begin{array}{l}\text { NMED } \\
\left(10^{-3}\right)\end{array}$ & $\begin{array}{l}\text { MRED } \\
\left(10^{-3}\right)\end{array}$ & $\begin{array}{l}\text { ER } \\
(\%)\end{array}$ & $\begin{array}{l}\text { Area } \\
\left(\mu m^{2}\right)\end{array}$ & $\begin{array}{l}\text { Power } \\
(\mu W)\end{array}$ & $\begin{array}{l}\text { Delay } \\
(n s)\end{array}$ \\
\hline 8884 & 2.04 & 0.16 & 3.28 & 445.6 & 58.86 & 0.77 \\
\hline 6664 & 7.68 & 0.64 & 4.39 & 410.4 & 54.10 & 0.66 \\
\hline 4444 & 31.10 & 2.59 & 8.89 & 385.6 & 50.49 & 0.56 \\
\hline 4422 & 32.18 & 2.70 & 25.26 & 369.5 & 47.72 & 0.56 \\
\hline 4321 & 59.29 & 5.16 & 37.57 & 360.3 & 45.99 & 0.56 \\
\hline 2222 & 117.91 & 10.46 & 32.32 & 351.6 & 74.29 & 0.40 \\
\hline
\end{tabular}

From the Table $\mathrm{V}$, we know that the error performance of SCPA becomes worse as the value of $w$ becomes smaller, but the corresponding power consumption area and computational delay become smaller. The value of $l$ in the design also can be adjusted. For example, in a 16-bit computation, one can choose $l_{3}, l_{2}, l_{1}, l_{0}=6,4,3,3$, increasing the bit width and prediction depth of the high-weighted sub-adders and decreasing the parameter values of the low-weighted adders, which can improve the statistical performance while keeping the circuit consumption of the adders as constant as possible.

\section{5. conclusion}

In this paper, an approximate computational adder structure based on the idea of the segmented adder is proposed. By separating the prediction depth and sub-adders parameters, a more flexible adder structure design idea is provided. Compared with similar adder designs,SCPA has a very clear advantage in terms of statistical error performance. Synthesis was performed at TSMC $65 \mathrm{~nm}$ CMOS process, and 
the results show that the SCPA has a moderate area and power performance. A good accuracy-power tradeoff is also achieved at 8-bit and 16-bit condition.

\section{Acknowledgments}

This work was supported by the Scientific research project in school-level(SZIIT2019KJ026) and Basic Research Discipline Layout Project of Shenzhen under Grant 2020B1515120004, Grant JCYJ20180507182241622, and Grant JCYJ20180503182125190.

\section{References}

[1] B. K. Mohanty et al.:"Area-Delay-Power Efficient CarrySelect Adder," IEEE Trans. Circuits Syst., II, Exp. Briefs 61 (2014) 418 (DOI:10.1109/TCSII.2014.2319695).

[2] B. Shao et al.:"Array-Based Approximate Arithmetic Computing: A General Model and Applications to Multiplier and Squarer Design," IEEE Trans. Circuits Syst. I, Reg. Papers 62 (2015) 1081 (DOI:10.1109/TCSI.2015.2388839).

[3] A. Raha et al.:"Input-Based Dynamic Reconfiguration of Approximate Arithmetic Units for Video Encoding," IEEE Trans. Very Large Scale Integr. (VLSI) Syst. 24 (2016) 846 (DOI:10.1109/TVLSI.2015.2424212).

[4] R. Ye et al.: "On reconfiguration-oriented approximate adder design and its application," ICCAD (2013) 48 (DOI:10.1109/ICCAD.2013.6691096).

[5] A. K. Verma et al.:"Variable latency speculative addition: a new paradigm for arithmetic circuit design," DATE (2008) 1250 (DOI:10.1145/1403375.1403679).

[6] T. Nomani et al.: "xUAVs: Towards Efficient Approximate Computing for UAVs-Low Power Approximate Adders With Single LUT Delay for FPGA-Based Aerial Imaging Optimization," IEEE Access 8 (2020) 102982 (DOI:10.1109/ACCESS.2020.2998957).

[7] G. Zervakis et al.: "Design Automation of Approximate Circuits With Runtime Reconfigurable Accuracy," IEEE Access 8 (2020) 53522 (DOI:10.1109/ACCESS.2020.2981395).

[8] J. Echavarria et al.: "Efficient Arithmetic Error Rate Calculus for Visibility Reduced Approximate Adders," IEEE Embedded Syst. Lett. 10 (2018) 37 (DOI:10.1109/LES.2017.2760922).

[9] D. Esposito et al.: "Variable Latency Speculative Parallel Prefix Adders for Unsigned and Signed Operands," IEEE Trans. Circuits Syst. I, Reg. Papers 63 (2016) 1200 (DOI:10.1109/TCSI.2016.2564699).

[10] S. Dutt et al:: "Analysis, Modeling and Optimization of Equal Segment Based Approximate Adders," IEEE Trans. Comput. 68 (2019) 314 (DOI:10.1109/TC.2018.2871096).

[11] J. Liang et al.: "New Metrics for the Reliability of Approximate and Probabilistic Adders," IEEE Trans. Comput. 62 (2013) 1760 (DOI:10.1109/TC.2012.146).

[12] C. Liu et al.: "An Analytical Framework for Evaluating the Error Characteristics of Approximate Adders," IEEE Trans. Comput. 64 (2015) 1268 (DOI:10.1109/TC.2014.2317180).

[13] S. Mazahir et al.: "Probabilistic Error Modeling for Approximate Adders," IEEE Trans. Comput. 66 (2017) 515 (DOI:10.1109/TC.2016.2605382).

[14] F. Frustaci et al.: "Energy-Quality Scalable Adders Based on Nonzeroing Bit Truncation," IEEE Trans. Very Large Scale Integr. (VLSI) Syst. 27 (2019) 964 (DOI:10.1109/TVLSI.2018.2881326).

[15] M. Pashaeifar et al.: "Approximate Reverse Carry Propagate Adder for Energy-Efficient DSP Applications," IEEE
Trans. Very Large Scale Integr. (VLSI) Syst. 26 (2018) 2530 (DOI:10.1109/TVLSI.2018.2859939).

[16] W. Xu et al.: "A Simple Yet Efficient Accuracy-Configurable Adder Design," IEEE Trans. Very Large Scale Integr. (VLSI) Syst. 26 (2018) 1112 (DOI: 10.1109/TVLSI.2018.2803081).

[17] N. Zhu et al:: "Design of Low-Power High-Speed Truncation-Error-Tolerant Adder and Its Application in Digital Signal Processing," IEEE Trans. Very Large Scale Integr. (VLSI) Syst. 18 (2010) 1225 (DOI:10.1109/TVLSI.2009.2020591).

[18] J. Hu et al.: "A high-accuracy approximate adder with correct sign calculation," Integration 65 (2019) 370 (DOI:10.1016/j.vlsi.2017.09.003).

[19] H. Jiang et al.:"A Review, Classification, and Comparative Evaluation of Approximate Arithmetic Circuits," J. Emerg. Technol. Comput. Syst. 13 (2017) 60 (DOI:10.1145/3094124)

[20] V. Gupta et al.: "Low-Power Digital Signal Processing Using Approximate Adders," IEEE Trans. Comput.Aided Design Integr. Circuits Syst. 32 (2013) 124 (DOI:10.1109/TCAD.2012.2217962).

[21] O. Akbari et al.: "RAP-CLA: A Reconfigurable Approximate Carry Look-Ahead Adder," IEEE Trans. Circuits Syst., II, Exp. Briefs $\mathbf{6 5} \quad$ (2018) 1089 (DOI:10.1109/TCSII.2016.2633307).

[22] P. Balasubramanian et al.: "Hardware Efficient Approximate Adder Design," TENCON 2018 2018 IEEE Region 10 Conference (2018) 0806 (DOI:10.1109/TENCON.2018.8650127).

[23] H. R. Mahdiani et al.: "Bio-Inspired Imprecise Computational Blocks for Efficient VLSI Implementation of Soft-Computing Applications," IEEE Transactions on Circuits and Systems I: Regular Papers 57 (2010) 850 (DOI:10.1109/TCSI.2009.2027626).

[24] P. Balasubramanian et al.: "Hardware Optimized and Error Reduced Approximate Adder," Electronics 8 (2019) 1212 (DOI:10.3390/electronics8111212).

[25] P. Balasubramanian et al:: "An Approximate Adder With a Near-Normal Error Distribution: Design, Error Analysis and Practical Application," IEEE Access 9 (2021) 4518 (DOI:10.1109/ACCESS.2020.3047651).

[26] Y. Kim et al.: "An energy efficient approximate adder with carry skip for error resilient neuromorphic VLSI systems," Proc. ICCAD (2013) 130 (DOI:10.1109/ICCAD.2013.6691108).

[27] A. B. Kahng et al.: "Accuracy-configurable adder for approximate arithmetic designs," Proc. 49th ACM/EDAC/IEEE Design Autom. Conf. (DAC) (2012) 820 (DOI:10.1145/2228360.2228509).

[28] J. Hu et al.: "A new approximate adder with low relative error and correct sign calculation," 2015 Design, Automation Test in Europe Conference Exhibition (DATE) (2015) 1449.

[29] F. Ebrahimi-Azandaryani et al.: "Block-Based Carry Speculative Approximate Adder for Energy-Efficient Applications," IEEE Trans. Circuits Syst., II, Exp. Briefs 67 (2020) 137 (DOI:10.1109/TCSII.2019.2901060).

[30] A. Dalloo et al.: "Systematic Design of an Approximate Adder: The Optimized Lower Part Constant-OR Adder," IEEE Trans. Very Large Scale Integr. (VLSI) Syst. 26 (2018) 1595 (DOI:10.1109/TVLSI.2018.2822278). 